MUZIKOLOŠKI ZBORNIK • MUSICOLOGICAL ANNUAL XL

UDK 78:271.3"16/18"

Ladislav Kačic

Kabinet für Slawistik der Slowakischen Akademie der Wissenschaften, Bratislava

Kabinet za slavistiko Slovaške akademije znanosti, Bratislava

\title{
Mitteleuropäische Kontexte der Franziskaner-Musikkultur im 17.-19. Jahrhundert
}

\section{Srednjeevropski okviri frančiškanske glasbene kulture v obdobju 17.-19. stoletja}

ZUSAMMENFASSUNG

Der Beitrag befaßt sich mit der Musik und Musikkultur in einigen Franziskanerprovinzen Mitteleuropas (Provincia S. Bernardini, Provincia S. Mariae in Hungaria, Provincia SS. Salvatoris, Provincia S. Wenceslai, Provincia S. Leopoldi, Provincia S. Ladislai, Provincia S. Joannis a Capistrano, Custodia S. Stephani, Provincia S. Antonii). Zuerst wird ein historischer Hintergrund dieser Provinzen im 17.-19. Jahrhundert skizziert. Im folgenden wird die Problematik der musikalischen Identität der Franziskaner in drei Problembereiche näher beschrieben: 1. Migration der Musiker (die war besonders im 17. Jahrhundert wichtig, als noch z. B. Kustodie des hl. Ladislaus zur Marianischen Provinz gehörte). 2. Das gemeinsame Repertoire war im 17. Jahrhundert von großer Wichtigkeit, es war spezifisch "mitteleuropäisch" (d. h. unterschiedlich z. B. von Italien u. ä.), im 18. Jahrhundert hat sich das Repertoire mehr und mehr zur Selbständigkeit entwickelt. Gemeinsam war für die Franziskanerprovinzen Mitteleuropas auch die Aufführungspraxis der Figuralmusik (kleine, "bescheidene" Besetzung, z. B. 1-stimmiger Chor mit Orgelbegleitung und 2 Trompeten). 3. Franziskanermusik und "lingua vernacula - ist auch ein bedeutender Beitrag zum Thema Identität, denn Franziskaner in Mitteleuropa haben vom 17. Jarhundert die sog. Volkssprachen gefördert. Es hat sich nicht nur in einer intensiven Entwicklung des Kirchenliedes geäußert, son-
PovZeTEK

Prispevek govori o glasbi in glasbeni kulturi v nekaterih frančiškanskih provincah Srednje Evrope (Provincia S. Bernardini, Provincia S. Mariae in Hungaria, Provincia SS. Salvatoris, Provincia S. Wenceslai, Provincia S. Leopoldi, Provincia S. Ladislai, Provincia S. Joannis a Capistrano, Custodia S. Stephani, Provincia S. Antonii). Najprej bo skicirano zgodovinsko ozadje omenjenih provinc v obdobju od 17. do 19. stoletja. V nadaljevanju bo v treh točkah podrobneje opisana problematika glasbene identitete frančiškanov: 1. Migracija glasbenikov je bila posebno pomembna v 17. stoletju, ko je npr. kustodija svetega Ladislava še pripadala marijanski provinci. 2. Skupni repertoar je bil v 17. stoletju velikega pomena. Bil je značilno "srednjeevropski" (to pomeni različen npr. od italijanskega ipd.). V 18. stoletju je postajal vedno bolj samostojen. Frančiškanskim provincam Srednje Evrope je bila skupna tudi izvajalska praksa figuralne glasbe (majhna, "skromna" zasedba, npr. enoglasni zbor s spremljavo orgel in dveh trobent). 3. Frančiškanska glasba in "lingua vernacula - je prav tako pomemben prispevek $\mathrm{k}$ vprašanju identitete, saj so frančiškani v Srednji Evropi od 17. stoletja naprej pospeševali t.i. ljudske jezike. To se ni kazalo samo $v$ intenzivnem razvoju cerkvene pesmi, temveč tudi v daljših kompozicijah (npr. arijah) na slovaška, nemška, madžarska idr. besedila. 
MUZIKOLOŠKI ZBORNIK - MUSICOLOGICAL ANNUAL XL

dern auch in längeren Kompositionen (z. B. Arien)

mit slowakischen, deutschen, ungarischen u. a.

Texten.

Für die richtige Wahrnehmung der Franziskaner-Musikkultur in Mitteleuropa ist es notwendig zuerst die Entwicklungsgeschichte, Peripetien und die historischen Zusammenhänge der mitteleuropäischen Franziskanerprovinzen zu erörtern. Obwohl Franziskaner im mitteleuropäischen Raum schon im 13. Jahrhundert zu Lebzeiten von hl. Franz von Assisi präsent waren ${ }^{1}$, konzentrieren wir uns auf ihre spätere Geschichte im Zeitraum vom 17. bis 19. Jahrhundert, als die Franziskaner nach dem stufenweisen Verdrängen der Türken aus dem mitteleuropäischen Raum einen mächtigen Aufschwung bis zu den Josephinischen Reformen erlebt haben.

Im Zusammenhang mit einem der größten Meilensteine in der franziskanischen Geschichte, der Teilung des Franziskanerordens 1517 auf zwei selbständige Orden Minoriten (Konventualen) und Franziskaner (Observanten) - hat das Generalkapitel in Burgos (1523) u. a. beschlossen, zwei selbständige Franziskanerprovinzen der Observanten im Königreich Ungarn zu gründen: Provincia S. Mariae in Hungaria und Provincia SS. Salvatoris. Diese zwei Provinzen wurden später, im 17. Jahrhundert, die Gründer anderer mitteleuropäischer Provinzen: im Rahmen der Marianischen Provinz entstand 1655 Custodia Slavoniae, die dank dem Fleiße von P. Paulus Jančić de Tauris OFM, einer der bedeutendsten Persönlichkeiten der Franziskaner in Mitteleuropa, ${ }^{2} 1661$ zur selbständigen Provincia S. Ladislai ernannt wurde. Aus der Salvatorianischen Provinz hat sich 1640 wiederum in Transylvanien Custodia S. Stephani getrennt, ${ }^{3}$ die aber erst 1729 zur Provinz erhoben wurde. (Ihre Interessen in dieser Kustodie, bzw. Provinz haben auch die Franziskaner aus Bosnien gewahrt.) Im 18. Jahrhundert entstanden im südslawischen Raum aus den ursprünglichen, streng observanten Provincia Bosnae Argentinae noch zwei neue Provinzen - 1757 Provincia S. Joannis a Capistrano und schon 1735 in Dalmatien Provincia SS. Redemptoris. Alle mitteleuropäischen Franziskanerprovinzen haben vor allem im 18. Jahrhundert eine intensive Entwicklung erlebt, wovon z. B. die Zahl der Klöster und Mitglieder Zeugnis ablegt: ${ }^{4}$

1 Die ersten Franziskaner hat schon das Generalkapitel 1217 nach Mitteleuropa gesandt. Nachrichten über die organisatorische Struktur sind jedoch erst etwa zehn Jahre später vorhanden - Custodia Strigoniensis, 1228.

2 P. Seraphinus Farkas OFM: Scriptores Ord. Min. S. P. Francisci Provinciae Hungariae Reformatae nunc S. Mariae. Posonii 1879, S. 22.

3 P. Odoricus Balázsovits OFM: Brevis historia conventuum Ordinis S. Franicsci Seraphici Reformatae Provinciae $S$. Mariae Hungariae. Posonii 1869, S. 19. Auch Fekete könyv. Az Erdélyi ferences kusztódia története, Kájoni János kézirata, 1684, hrsg. von Kornél Szovák. Szeged 1991.

4 P. Heribert Holzapfel OFM: Handbuch der Geschichte des Franziskanerordens. Freiburg im Breisgau 1909, S. 415-417. 
MUZIKOLOŠKI ZBORNIK • MUSICOLOGICAL ANNUAL XL

Tabelle Nr. 1

\begin{tabular}{|l|l|l|l|}
\hline Provincia & ca. 1680 & $\mathbf{1 7 0 0}$ & $\mathbf{1 7 6 2}$ \\
\hline S. Mariae in Hungaria & $18 / 361$ & $24 / 350$ & $28 / 856$ \\
\hline Sanctissimi Salvatoris & $18 / 325$ & $23 / 352$ & $32 / 931$ \\
\hline Sancti Ladislai Regis & $9 / 145$ & $17 / 166$ & $17 /[?]$ \\
\hline S. Bernardini (Wiener Prov.) & $22 / 484$ & $24 / 580$ & $27 / 871$ \\
\hline S. Leopoldi (Tiroler Provinz) & $13 / 253 ?$ & $17 / 333$ & $18 / 489$ \\
\hline S. Antonii (Bayerische P.) & $20 / 520$ & $27 / 582$ & $37 / 1016 !$ \\
\hline S. Wenceslai (Bohemische P.) & $22 / 493$ & $29 / 681$ & $22 / 413$ \\
\hline Carniolae & & $16 / 293$ & $18 / 444$ \\
\hline S. Stephani (Transylvania) bis 1729 Custodia & $3 / 42$ & $4 / 53$ & $13 / 301$ \\
\hline Bosnae Argentinae & $18 / 426$ & $23 / 420$ & $23 /[?]$ \\
\hline Bosnae Croatiae (bis 1688) & $5 / 180$ & & \\
\hline S. Ioannis Capistrani & - & - & $?$ \\
\hline SS. Redemptoris in Dalmatia & & & \\
\hline
\end{tabular}

Alle diese Provinzen kann man natürlich aus dem Aspekt der Nationalität nicht als homogehe Einheiten betrachten. Es war eher umgekehrt, z. B. in der Marianischen Provinz wurden 1727-1735 in den Nationalitätenstreit der Deutschen und Ungarn als dritte große Gruppe auch Slowaken und Kroaten (zusammen) als eine "Natio slavica" einbezogen. ${ }^{5}$ Während der Josephinischen Reformen wurde die Zahl der Klöster und der Franziskaner stark reduziert, z. B. Marianische Provinz hatte an der Wende des 18. Jahrhunderts nur 20 Klöster und 368 Franziskaner; ${ }^{6}$ noch schlimmer war es in Österreich. Von der Wiener Provinz des hl. Bernardin sind in den ersten Jahrzehnten des 19. Jahrhunderts nur 4 Klöster geblieben (Wien, St. Pölten, Maria Enzersdorf, Maria Lanzendorf), die 1825 der Kapistranischen Provinz zugeteilt wurden. ${ }^{7}$ Das "nachaufklärerische", säkularisierte 19. Jahrhundert bedeutete eindeutig den Verfall des Franziskanertums in Mitteleuropa und damit auch den Verfall der Musik dieses Ordens. Wenn wir also die musikalische Identität Mitteleuropas am Beispiel der Franziskaner näher beobachten wollen, ist es notwendig die Situation, wie sie in diesen kurzen allgemeingeschichtlichen Darstellung skizziert ist, im Auge zu behalten.

1. Migration der Franziskaner-Musiker. Weil in der 1. Hälfte des 17. Jahrhunderts mitteleuropäische Franziskaner-Mutterprovinzen noch größer als später waren

\footnotetext{
5 Vgl. näher Ladislav Kačic: P. Marcus Repkovič OFM a národnostný spor františkánov mariánskej provincie v rokoch 1727-1735. In: Slavica Slovaca, Bratislava 1997, Jg. 32, Nr. 2, S. 59-71. Einen ähnlichen Streit gab es schon im 17. Jahrhundert in der Salvatorianischen Provinz zwischen den ungarischen und slowakischen Mitbrüdern. Vgl. dazu P. Laetus Danišovič OFM: Dejiny minoritov I. - Osvietenská historiografia Rádu františkánskeho v našich krajinách. Bratislava 1934, S. 93-99.

6 P. Arnold Magyar OFM: 340 Jahre Franziskaner in Güssing (1638-1978). Güssing 1980, S. 24.

7 Ebenda, S. 27.
} 
und ihre Klöster sich auf einem relativ großem Territorium befanden (z. B. Marianische Provinz - die heutige Slowakei, Ungarn, Burgenland, Slowenien), umfasste die Migration der Musiker - Organisten, Regenschori - damals ein viel breiteres Territorium als später. So z. B. haben dieselben Musiker (Organisten, Directores chori) der Marianischen Provinz sowohl in Bratislava/Preßburg oder Trnava/Tyrnau, als auch in Zagreb, Varaždin, Križevici, Krapina und Ormož gewirkt: P. Casimirus Vogler (er war auch Komponist) in Varaždin (1647), Križevici (1648-49) und Zagreb (1650-55), P. Emericus IsaAc in Križevici (1646), Ormož (1647), Varaždin (1649, 1652-55) und Krapina (1650), P. Athanasius Naßer in Varaždin (1646, 1650-51), Križevici (1647), Ormož (1649 und Zagreb (1652).

Die Migration der Franziskanermusiker zwischen den Provinzen war aber eher eine Ausnahme. So z. B. ist einer der bedeutendsten Franziskaner Komponisten des 17. Jahrhunderts in Mitteleuropa, der aus Mähren stammende P. Franciscus Vogler (um 1623-1688) im Jahre1668 aus der Marianischen Provinz in die Salvatorianische übergetreten. ${ }^{8}$ Ein anderes Beispiel stellte im 18. Jarhundert P. Dismas Grapmayr (1721-1808), ebenfalls ein guter Komponist, dar. Er war ursprünglich Mitglied der Österreichischen Provinz des hl. Bernardin und trat 1761 (als "in mente turbatus") aufgrund einer Intervention des Ordensgeneralministers zur Marianischen Provinz über. ${ }^{9}$ Diese Tatsachen, d. h. die Migration der Musiker zwischen den Provinzen, hatten konkrete Folgen in der Musik, vor allem im Repertoire.

2. Das gemeinsame Repertoire. Obwohl die Franziskanerprovinzen in Mitteleuropa relativ geschloßene organisatorische Einheiten bildeten, ähnlich auch im augeführten Musikrepertoire ${ }^{10}$ war im 17. Jahrhundert dieses Repertoire noch zum Großteil gemeinsam, wie es am Beispiel der Messe folgende Tabelle (S.118) zeigt:

* Diese Messe ist zum Teil gregorianisch, sie ist nicht eindeutig einzuordnen (s. Pál Richter: Organo-Missale-Musical Relationships of a Franciscan Manuscript in the $17^{\text {th }}$ Century, in: Musik der Geistlichen Orden in Mitteleuropa zwischen Tridentinum und Josephinismus. hrsg. von Ladislav Kačic, Bratislava 1997, S. 140).

Auf den ersten Blick kann man hier einige deutlichen Merkmale erkennen. Es fällt ins Auge vor allem die große Zahl gemeinsamer Messen in zwei benachbarten Provinzen auf, z. B. in der Wiener Provinz des hl. Bernardin und der Marianischen Provinz, ebenso in bezug auf die Böhmische Provinz des hl. Wenceslaus (inklusive Messen der "nicht«-franziskanischen Komponisten! ${ }^{11}$ usw. Aber einige Messen, wie z. B. jene von P. Eusebius Schreiner (ME I/186) und andere als "Viennensis" (bzw.

8 Näheres über ihm s. P. Vševlad Gajoš OFM: Dva hudobné zborniky zo 17. storočia. In: Musicologica slovaca I/2, Bratislava 1969, S. 301, sowie Ladislav Kačic: Missa franciscana der Marianischen Provinz im 17. und 18. Jahrhundert. In: Studia Musicologica Academiae Scientiarum Hungaricae 33, Budapest 1991, S. 29.

9 Seine fast "romanhafte" (kurz zusammengefaßte) Lebensgeschichte siehe ebenda, S. 27-28.

${ }^{10}$ Ausführilicher zu dieser Problematik s. Ladislav Kačic: Repertorie und Aufführungspraxis der Kirchenmusik in den Franziskanerprovinzen Mitteleuropas im 17.-18. Jahrhundert. In: Musicologica Istropolitana I, Universitas Comeniana Facultas Philosophica Bratislava, hrsg. von Marta Hulková und =ubomír Chalupka, Bratislava 2002, S. 53-102.

${ }^{11}$ Im untersten Teil der Tabelle - P. Bernardus Artophaeus OFMConv, Alexander Hrdý, P. Adalbertus Pelikán SP. Diese Messen wurden Bestandteil der Repertoires der Wiener Provinz des hl. Bernardin. 
"Viennense") bezeichnete Werke, waren für alle mitteleuropäischen Provinzen gemeinsam. Diese oft vorkommende Werke, zu denen ME I/183, I/185, I/187, I/189, I/190, aber z. B. auch Requiem von P. Quirinus Himmer, ME I/101 und eine Messe von P. Eduard Panholzer aus Tirol (ME I/123) gehören, beweisen, daß die Wiener Franziskanerprovinz in Mitteleuropa eine zentrale Rolle spielte. Aus dieser Provinz sind auch die ersten im spezifisch Franziskanischen Stil komponierte Figuralmessen für einstimmigen Mönchschor mit Orgelbegleitung und nur mit zwei Trompeten aus der Zeit um die Wende des 17. Jahrhunderts in die Nachbarprovinzen gelangt (ME I/140, ME I/128 u. a.).

Dieses kurz beschriebene gemeinsame Repertoire war also spezifisch für die Franziskaner in Mitteleuropa und unterscheidet sich grundsätzlich von anderen Regionen Europas (z. B. Italien). Spezifisch "mitteleuropäisch" war auch die Aufführungspraxis der Franziskanischen Figuralmusik: im Grunde geht es um den 1-stimmigen (im 18. Jahrhundert höchstens 2-stimmigen $\mathrm{C}+\mathrm{B}$ ) Chor mit Orgelbegleitung, dazu zuerst 2 Trompeten (oder Hörner), und letztendlich auch Streichinstrumente (das sog. Kirchentrio). Diese Praxis ist in allen mitteleuropäischen Provinzen quellenmäßig belegt, obwohl Stimmhefte für Instrumente nur aus der Wiener Provinz des hl Bernardin, sowie aus der Marianischen und Bayerischen Provinzen überliefert sind. ${ }^{12}$ Auch die spätere Praxis der einfachen 2-, (höchstens 3-) stimmigen Vokalbesetzung mit Orgelbegleitung war für alle Franziskanerprovinzen Mitteleuropas gemeinsam. ${ }^{13}$

Im 18. Jahrhundert hat sich das Musikrepertoire in den mitteleuropäischen Franziskanerprovinzen nach und nach verselbständigt. Nach heutigen Erkentnissen pflegte jede Provinz mehr und mehr nur ihre "eigene" Musik, $d . h$. das Schaffen der eigenen Provinzkomponisten gewann das Übergewicht. Im 18. Jahrhundert gibt es ein stark reduziertes gemeinsames Repertoire fast ausschließlich nur in je zwei Provinzen, nur sehr wenige Werke haben allgemeine Verbreitung gefunden. $\mathrm{Zu}$ ihnen gehören vor allem fast hundertprozentig anonym überlieferte Messen des weltlichen Organisten des Wiener Franziskanerklosters FerdinAND STEINER ( $†$ 1750): ${ }^{14}$ eine seiner C-dur-Messen wurde Bestandteil des Repertoires in der Provinz des hl. Bernardin, in der Marianischen, Böhmischen, Tiroler Provinzen, sowie in der Kapistraner Provinz, u. zw. im vom P. Josephus Maria Cordans OFM zusammengestellten und vom Provinzial, sowie Generaldeffinitor P. Josephus Janković OFM 1750 streng angeordnetem Meßrepertoire $^{15}$ (als Missa S. Didaci). Zu diesem Repertoire gehörte auch eine ebenfalls nur anonym überlieferte C-dur-Messe des in Tirol wirkenden weltlichen Komponisten Giovanni Abondio Grotti, die nicht nur zum Stammrepertoire in Tiroler Provinz des hl. Leopold, sondern auch zum Repertoire der Wiener Provinz (ME I/167), sowie der Marianischen und Kapistranischen (Missa S. Clarae) Provinzen gehörte. Die Verfasser der weiteren zweier Messen des gemeinsamen mitteleuropäischen Franziska-

\footnotetext{
${ }^{12}$ Mehr darüber s. Ladislav Kačic: Repertorie und Aufführungspraxis, a. a. O., S. 85-98.

${ }^{13}$ Ebenda, vgl. auch Vjera Katalinić: Katalog muzikalija u franjevačkom samostanu u Omišu, Zagreb 1991.

${ }^{14}$ Näheres über ihm s. Ladislav Kačic: Missa franciscana, a. a. O., S. 27.

${ }^{15}$ Vgl. Ladislav Kačic: Repertoire und Aufführungspraxis, a. a. O., S. 68, sowie Vjera Katalinić: Von Jesuiten zu Franziskanern - Kroatische geistliche Musikkultur im 18. Jahrhundert. In: Musik der geistlichen Orden in Mitteleuropa zwischen Tridentinum und Josephinismus, hrsg. von Ladislav Kačic, Bratislava 1997, S. 101.
} 
MUZIKOLOŠKI ZBORNIK • MUSICOLOGICAL ANNUAL XL

Tabelle Nr. 2: Anmerkung: Die in drei und mehreren Provinzen auffindbare Messen sind in Halbdick-, die Figuralmessen in Kursivschrift aufgezeichnet, wobei hier bemerkt werden muß, daß zwischen der Figuralmesse und der typischen Franziskaner Missa choralis keine "eindeutige" Trennungslinie existiert. Dies betrifft auch Messen auf verschiedene gregorianische Canti firmi oder Kirchenlieder.

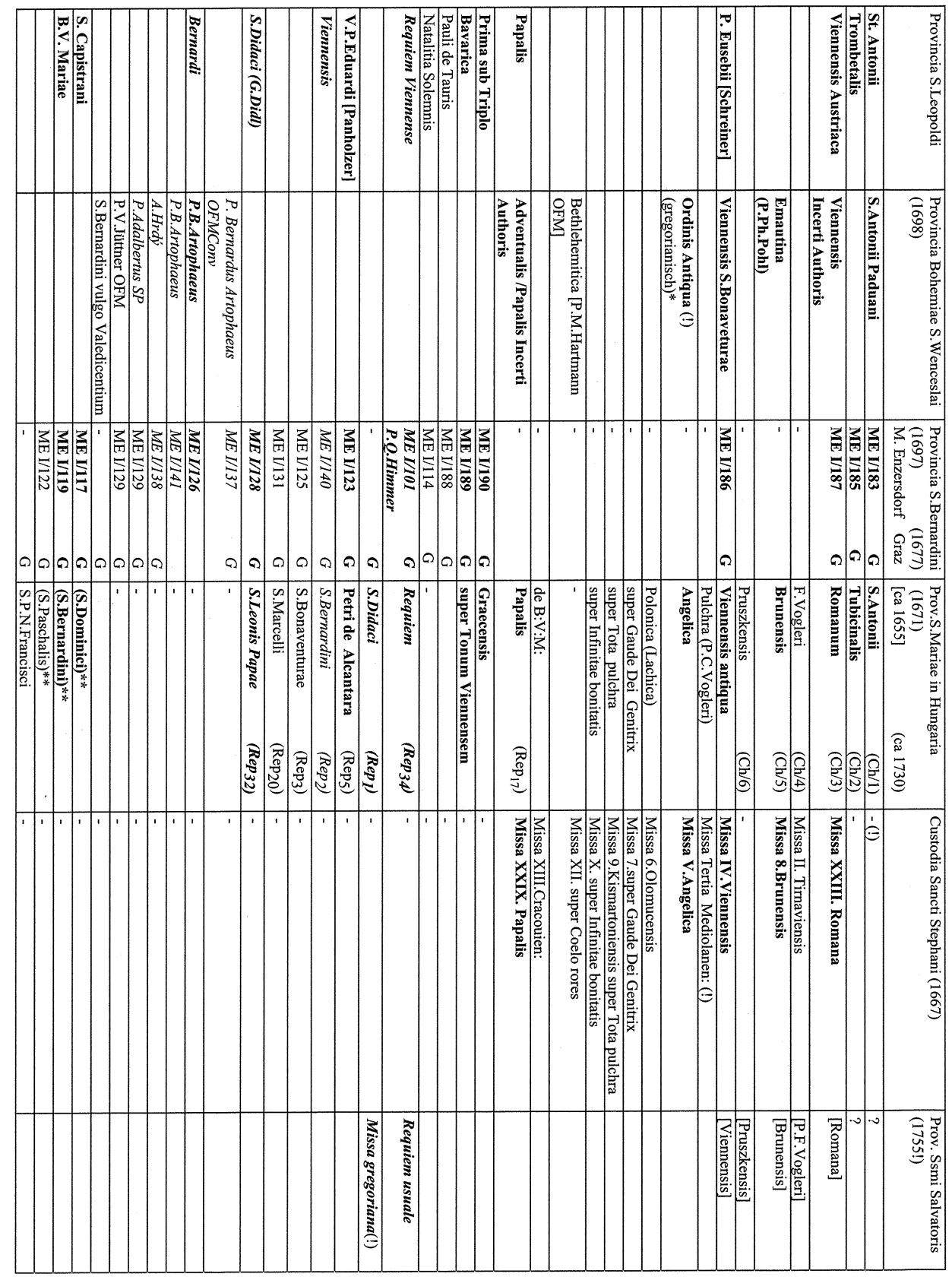


nerepertoires des 18. Jahrhunderts - ME I/134 und ME I/172 - ist es nicht gelungen zu identifizieren (sie bleiben vorläufig nur als "Austriaca", $d$. h. halbanonym bekannt). Viele solche Kompositionen, die man als "halbanonym" bezeichnen darf, gehörten zum Stammrepertoire von mehr als zwei mitteleuropäischen Franziskanerprovinzen, sie trugen oft auch im 18. Jahrhundert noch "topographische" Bezeichnungen: Missa Viennenesis, Tota pulchra Olomoucense, Lytaniae Bavaricae, Missa Bohemica, Sacrum Slavonicum, Lytaniae Carniolicae, Missa Croatica usw. Solche Titel beweisen, daß das Repertoire auch im 18. Jarhundert noch migrierte, wenn man auch den konkreten Autor nicht genau wußte (er war für die Franziskaner keinesfalls bedeutend, bzw. interessant $)^{16}$. Zwischen anonymen Werken, die im 18. Jahrhundert in mehreren Franziskanischen Provinzen Mitteleuropas gespielt wurden, kann man nicht nur Ordenskomponisten, wie z. B. P. Engelbertus Katzer (1719-1779) oder P. Adrianus Damian (1708-1750) aus der Wiener Provinz, oder P. Pantaleon RošKovskÝ (1734-1789) und P. Gaudentius DetTelbach (1739-1818) aus der Marianischen Provinz u. v. a. identifizieren, sondern manchmal auch allgemein bekannte und augeführte Komponisten, wie den Herzogenburger Augustinerchorherren Johann GEORg Donberger oder den weltlichen Priester aus Bozen CANDidus FarteluI. ${ }^{17}$

3. Franziskanermusik und „ingua vernacula «. Franziskaner gehörten immer zu den größten Förderern der Volkssprachen, es genügt an dieser Stelle nur den Gründer des Ordens und seine "Laudes creaturarum" (Canticum de sol"), oder den großen Dante Alighieri, einen Franziskaner-Tertiaren, zu nennen. Es ist bekannt, daß Franziskaner vom Anfang an große Bedeutung den Volkssprachen in Seelsorge beigemessen haben. In mitteleuropäischen Provinzen hatten z. B. einzelne Klöster im 17.-19. Jahrhundert spezielle Prediger (slowakische, deutsche, ungarische, kroatische usw.) für alle Sprachen der Bevölkerung in der Umgebung des gegebenen Klosters. Mehrere namhafte slowakische Musiker und Komponisten aus dem Franziskanerorden waren zugleich bedeutende Prediger, z. B. P. Paulinus Bajan, P. Nobertus Foit, P. Marcus Repkovič, P. Georgius Zrunek u. a. Etwas Ähnliches wie für die Redekunst gilt auch für die Franziskanermusik. Seit dem 17. Jahrhundert hat vor allem das Kirchenlied bei den Franziskanern Mitteleuropas eine große Entwicklung erfahren. ${ }^{18}$ Es sind an dieser Stelle z. B. viele franziskanische slowakische Gesangbücher aus der Marianischen und Salvatorianischen Provinzen zu nennen: schon seit den 1660er Jahren des 17. Jh. hat P. EDmund BEHovč die sog. Handschriftliche Gestalt des Cantus Catholici ${ }^{19}$ bei seiner Missionstätigkeit in der damals zum Großteil protestantischen Städten und Ortschaften der heutigen Südwest-Slowakei verwendet. Später im 18.

\footnotetext{
${ }^{16}$ Zum Problem der Anonymität des "Opus franciscanum" s. Ladislav Kačic: Opus franciscanum v zápise a zvukovej podobe. In: Slovenská hudba, 18. Jg., 1992, Nr. 1, S. 136-145.

${ }_{17}$ Ladislav Kačic: Repertoire und Aufführungspraxis, a. a. O., S. 71 u. 85.

${ }^{18}$ Über das deutsche und ungarische Kirchenlied s. näher Pál Richter: Die Verwerdung der Kirchenlieder in der Franziskaner Quellen (17.-18. Jahrhundert). In: Gegenreformation und Barock in Mitteleuropa / in der Slowakei, hrsg. von Ladislav Kačic; Bratislava 2000, S. 207-226.

${ }^{19}$ Näheres über diese wichtige Quelle s. Peter Ruščin: Duchovné piesne notovaných slovenských katolíckych spevníkov 17. storočia (PhD.), Bratislava 1999, sowie Peter Ruščin: Najstarši rukopisný slovenský katolícky spevnik. In: Slavica Slovaca, Bratislava 1999, Jg. 34, Nr. 2, S. 106-123.
} 
MUZIKOLOŠKI ZBORNIK • MUSICOLOGICAL ANNUAL XL

Jahrhundert hat allein P. PAULINUS BAJAN (1721-1792) fünf Gesangbücher mit vornemlich slowakischen und teilweise auch leteinischen Texten geschrieben: Jubilus Cordis III (1749), Beckovský slovenský spevník (1758), Promptuarium Hebdomadae Sanctae (1758), Promptuarium chorale (1780) und Skalický slovenský spevník (1783). ${ }^{20}$ Ähnlich lässt sich es bei anderen seinen Mitbrüdern konstatieren. ${ }^{21}$ Diese Gesangbücher beinhalten nicht nur Kirchenlieder, sondern oft auch Arien in slowakischer Sprache, oder auch lateinische und lateinisch-slowakische Kompositionen (mit sog. makaronischem Text). Zu den originellsten Schöpfungen vielleicht nicht nur der Franziskanermusik in Mitteleuropa gehören zwei lateinisch-slowakische, früher irrtümlich P. Edmund Pascha OFM zugeschriebene Weihnachtsmessen von P. GEORGIUs ZRuneK (1736-1789) aus seiner Harmonia pastoralis (1766), die auch in lateinisch-ungarischen Versionen (aus dem Jahr 1767) existierten. ${ }^{22}$ In diesem Zusammenhang ist auch die Anordnung des Deffinitoriums der Salvatorianischen Provinz aus dem Jahre 1791 interessant: "ut cantus lingua vernacula in tota Alma Provincia uniformiter cantetur. ${ }^{23}$ Nicht nur in den Wiener und Tiroler Provinzen sind viele deutsche Kirchenlieder, ${ }^{24}$ als auch Arien usw. in deutscher Sprache vorzufinden, sondern in der national stark gemischten Marianischen Provinz sind solche Werke in slowakischer, deutscher und ungarischer Sprache übeliefert (neben Kirchenliedern noch Strophen- und DaCapo-Arien). Von der Sprache her sind ebenfalls Gesangbücher des 19. Jahrhunderts aus dieser Provinz bezeichnend: Kirchenlieder (1847) und vor allem Nábožné katolické pesničky (1848) des Fr. Coecilanus Plihal beinhalten sowohl slowakische (50\%), als auch deutsche (20\%) und ungarische (30\%) Kirchenlieder. ${ }^{25}$ Fr. C. Plihal wirkte nämlich in der "dreisprachigen" Stadt Preßburg. Er ist aber nur ein typischer stellvertretender Beispiel für viele seine Vorgänger und Zeitgenossen im Franziskanerorden in allen Provinzen Mitteleuropas.

\footnotetext{
${ }^{20}$ Vgl näher P. Vševlad Gajdoš OFM: Hudobné zborníky Pavlina Bajana. In: Musicologica slovaca III, Bratislava 1971, S. 181-225, sowie Ladislav Kačic: P. Paulín Bajan - františkánsky hudobnîk a kazate>. In: P. Paulín Bajan OFM (1721-1792) a slovenská hudba, literatúra, jazyl 18. storočia, hrsg. von Ladislav Kačic, Bratislava 1992, S. 51-64.

${ }^{21}$ Von P. Marcus Repkovič sind 2 umfangreiche lateinisch-slowakische Gesangbücher überliefert, von P. Macarius Hargaš ebenfalls 2, von P. Georgius Zrunek (zusammen mit P. Edmund Pascha) 1 usw.

${ }^{22}$ P. Georgius Zrunek OFM: Missa I pro Festis Natalitiis (ex Harmonia pastoralis), hrsg. von Ladislav Kačic, Bratislava 1993.

${ }^{23}$ Ernest Zavarský: Príspevok $k$ dejinám hudby v Kremnici v rokoch 1651-1800. In: Hudobný archív 3, Martin, S. 340.

${ }^{24}$ Vgl. Pál Richter: Die Verwendung der Kirchenlieder, a. a. O., passim.

${ }^{25}$ Ladislav Kačic: hudba františkánov na Slovensku v 19. storočí. In: Duchovná hudba v 19. storočí (Bibliotheca Musicae Neosoliensis, Bd. 2), hrsg. von Jana Lengová, Banská Bystrica 1995, S. 57-63.
} 